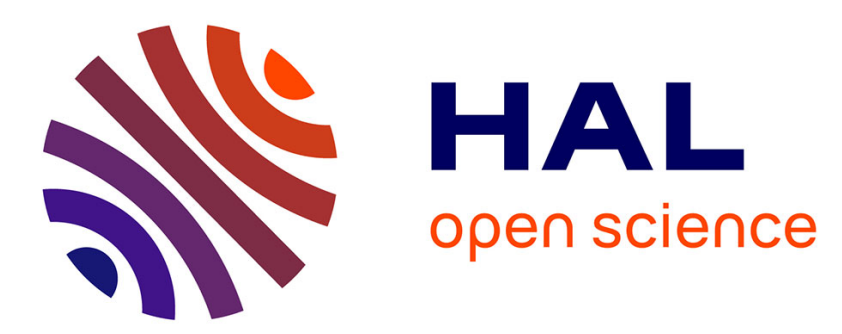

\title{
Design and fabrication of an asymmetric twin-core fiber directional coupler for gain-flattened EDFA
}

\author{
B. Nagaraju, Michèle Ude, Stanislaw Trzesien, Bernard Dussardier, Ravi K. \\ Varshney, Gérard Monnom, Wilfried Blanc, Bishnu P. Pal
}

\section{- To cite this version:}

B. Nagaraju, Michèle Ude, Stanislaw Trzesien, Bernard Dussardier, Ravi K. Varshney, et al.. Design and fabrication of an asymmetric twin-core fiber directional coupler for gain-flattened EDFA. Asia Communications and Photonics Conference, Nov 2011, SHANGHAI, China. pp.8307-17. hal00643637

\section{HAL Id: hal-00643637 https://hal.science/hal-00643637}

Submitted on 22 Nov 2011

HAL is a multi-disciplinary open access archive for the deposit and dissemination of scientific research documents, whether they are published or not. The documents may come from teaching and research institutions in France or abroad, or from public or private research centers.
L'archive ouverte pluridisciplinaire HAL, est destinée au dépôt et à la diffusion de documents scientifiques de niveau recherche, publiés ou non, émanant des établissements d'enseignement et de recherche français ou étrangers, des laboratoires publics ou privés. 


\title{
Design and fabrication of an asymmetric twin-core fiber directional coupler for gain-flattened EDFA
}

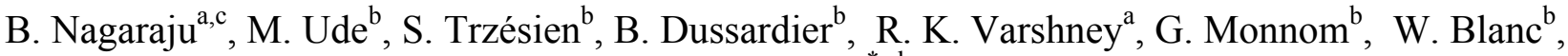 \\ and B. P. Pal ${ }^{* a, b}$

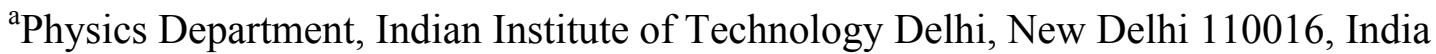 \\ ${ }^{b}$ Laboratoire de Physique de la Matière Condensée - Université de Nice Sophia Antipolis UMR \\ CNRS 6622, - Parc Valrose, F 06108 NICE Cedex 2, France \\ ${ }^{\mathrm{c}}$ Sterlite Technologies Ltd., Aurangabad, Maharashtra 431136, India
}

\begin{abstract}
Fiber directional couplers made of highly asymmetric twin-cores (ATC), one of which is doped with erbium, are designed to achieve an inherently gain flattened erbium doped fiber amplifier (EDFA). The refractive index profiles of the fibers as well as the spacing between the two cores were carefully designed to achieve a targeted gain with low gain excursions across the C-band. One of the designs yielded a theoretical median gain $\sim 38 \mathrm{~dB}$ with an excursion within \pm 1 $\mathrm{dB}$. In order to suite fabrication of such an inherently gain flattened EDFA by the MCVD fiber preform fabrication process, the design had to be modified and a more modest target of about $20 \mathrm{~dB}$ was set with excursion below $\pm 1.5 \mathrm{~dB}$ for metro-centric applications. It involved preparation of two independent preforms, which required selective polishing of the cladding from one side by a certain amount to meet the required nominal separation between the two cores set at the design stage of the fiber. Several intricate operations were required to implement the fiber drawing step from the two assembled preforms. Preliminary characterization of the fabricated fiber shows filtering of ASE peak through selective wavelength coupling from Er-doped core to un-doped core.
\end{abstract}

Keywords: Erbium doped fiber amplifiers, Gain flattened EDFA, Modified chemical vapor deposition technique

\section{INTRODUCTION}

Erbium doped fiber amplifiers (EDFA) find wide scale applications in various medium to long-haul optical communication networks including metro networks because of their broad gain, low noise and high-speed response. Due to broad gain spectrum these find extensive applications in dense wavelength division multiplexing (DWDM) systems. However, the non-uniform gain spectrum in conjunction with the saturation effects of EDFAs could cause degradation of the optical signal-to-noise ratio (OSNR) to unacceptable values in systems consisting of cascaded chains of EDFAs. These features could limit the usable bandwidth of EDFAs and hence the data transmission capacity of the system. Accordingly various schemes of gain equalizing filters have evolved in the literature. From this perspective, an intrinsically gain flattened EDFA would cut down the cost on the gain equalization filter head. Such inherently gain equalized EDFAs are attractive for metro-centric networks because low cost components and less number of components (to cut down the network maintenance cost) have been a major driver in a metro network design. Exploitation of a wavelength filtering mechanism inherent in a co-axial dual-core fiber design scheme to realize a gain flattened EDFA was reported by us earlier [1]. Laming et al were possibly first to propose in 1993 a scheme for gain equalization utilizing a twin core amplifier [2]. In their design, the equalized gain bandwidth was quite narrow centered on the gain peak wavelength. Later, other authors [3] proposed modified scheme and logistic for better and efficient use of a twincore fiber directional coupler concept to achieve flat gain with a gain excursion of $0.7 \mathrm{~dB}$ over $30 \mathrm{~nm}(1525-1555 \mathrm{~nm})$ in the C-band. It consisted of a twin-core Erbium doped fiber (EDF) in which only one of the cores (core 1) is doped with $\mathrm{Er}^{3+}$ and 980/1550 nm power through a WDM coupler was launched and retrieved from the core 1 only. The excess power at wavelength around $1530 \mathrm{~nm}$ due to non-uniform gain characteristic of typical EDFAs was coupled strongly to the undoped core 2 . However, a drawback with this idea was that the absolute gain levels showed significant variation

\footnotetext{
*bppal@physics.iitd,ernet.in: phone 91112659 1331; fax 91112658 1114; work was partially supported at the India-end by a UKIndia Education and Research Initiative (UKIERI) project. BPP acknowledges support by CNRS for hosting his short visit to LPMC.
} 
with change in input pump power and hence such a configuration would be quite susceptible to transient changes in the pump and signal powers, and thus would require some type of complex gain-clamping arrangement. Moreover, no theoretical analysis is given regarding the optimization of the twin core fiber, which is important for fabricating such fiber. In this paper, we propose a new asymmetric twin-core (ATC) gain flattened EDF design that is based on filtering action induced by resonant power coupling from doped core to un-doped core.

\section{FIBER DESIGN}

We consider a highly asymmetric twin core (ATC) fiber, in which the $\mathrm{Er}^{3+}$-doped core is relatively large with a smaller core-cladding refractive index contrast and the un-doped core, of a larger index contrast, is relatively narrow as shown in Fig. 1.

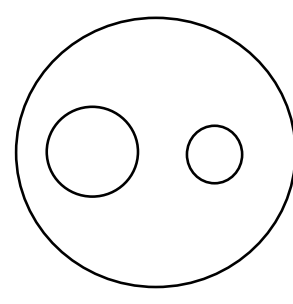

(a)

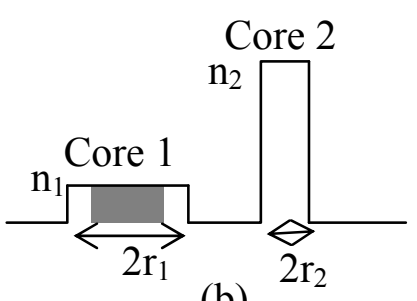

(b)

Fig. 1 Schematic diagram of (a) cross sectional view and (b) corresponding RIP of the twin core fiber.

The fiber parameters were carefully chosen such that the fundamental modes corresponding to the isolated fibers are phase-matched at a wavelength of nearly $1530 \mathrm{~nm}\left(\lambda_{\mathrm{p}}\right)$. It is well known that in an asymmetric directional coupler, the supermodes of the coupler correspond to almost the individual fiber modes away from $\lambda_{\mathrm{p}}$. Below $\lambda_{\mathrm{p}}$, effective mode index ( $\left.n_{\text {eff }}\right)$ of the fundamental mode of the composite structure would lie very close to that of core 1 while above $\lambda_{\mathrm{p}}$, its $n_{\text {eff }}$ lies close to that of core 2 and as one crosses $\lambda_{\mathrm{p}}, n_{\text {eff }}$ of the two normal modes vary very rapidly. The variation of effective modal index $\left(\mathrm{n}_{\text {eff }}\right)$ of two normal modes in combination with the doped and un-doped core modes with wavelength is shown in Fig. 2. This asymmetric directional coupler structure essentially acts like a wavelength filter with dip in the transmittance versus $\lambda$ curve around $\lambda_{\mathrm{p}}$. With these physical features in mind, at the design stage we optimized the fiber parameters, doping level and signal and pump powers in order to minimize the gain excursion in the gain spectrum of the EDFA.

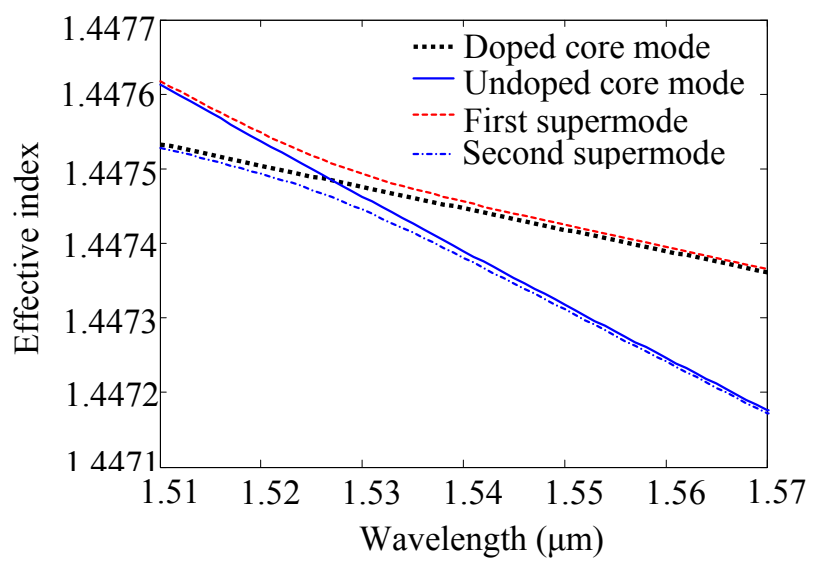

Fig.2.Variation of effective refractive indices of individual core modes, and the super modes of the ATC fiber. 


\section{THEORY AND DESIGN OPTIMIZATION METHODOLOGY}

The gain characteristics of this ATC EDF were modeled through the standard three-level rate equation model [4]. We have assumed forward pumping at the wavelength of $980 \mathrm{~nm}$. The nonlinearly coupled differential equations, which govern the propagation of ASE, signal and pump power along the length of the fiber, were solved using fourth-order Runge-Kutta method under the adaptive step size approach. Modal intensities of supermodes required in the numerical simulation were obtained using Coupled Mode Theory [5]. When the individual cores are weakly coupled and single moded in isolation from each other, electric field $\Psi_{ \pm}(\mathrm{x}, \mathrm{y})$ of the normal modes can be approximated as-

$$
\Psi_{ \pm}(x, y)=\Psi_{1}(x, y)+a_{ \pm} \Psi_{2}(x, y)
$$

Plus and minus correspond to two supermodes, namely first and second supermodes of the combined structure. $\Psi_{1}(\mathrm{x}, \mathrm{y})$ and $\Psi_{2}(\mathrm{x}, \mathrm{y})$ represent the transverse mode field pattern of fiber-1 and 2, respectively in absence of other. We have considered the excitation of both supermodes in our modeling. Variation of excitation efficiency of the fundamental and first higher-order modes with wavelength is shown in Fig. 3. As can be seen from this figure, the excitation efficiency of two modes varies rapidly around $\lambda_{\mathrm{p}}$. For optimization of fiber parameters, as a first step, we had chosen first fiber core's parameters in such a way that it supports single-mode. Next we calculated the parameters of second core in

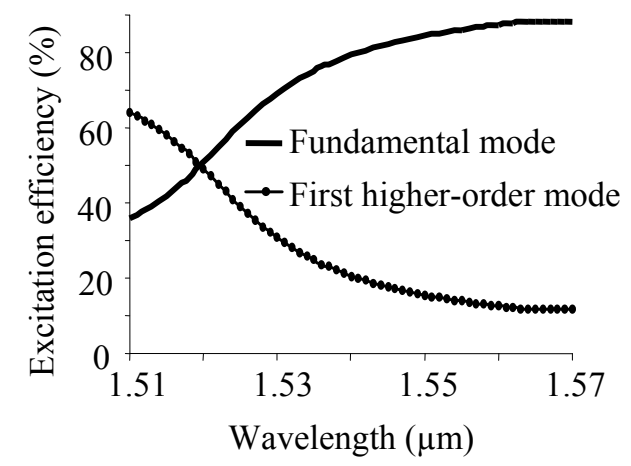

Fig. 3: Variation of excitation efficiencies of the fundamental and first higher-order modes when the EDF is spliced with a SMF-28 fiber.

such a way that it is also single moded and the propagation constants of the two cores match at the $\lambda_{\mathrm{p}} \sim 1530 \mathrm{~nm}$. However, for achieving gain flattening, $\lambda_{\mathrm{p}}$ as well as slopes of two curves for $n_{\mathrm{eff}} \mathrm{vs} \lambda$ corresponding to the individual core modes needs to be optimized. After obtaining this initial set of parameters, separation between the two cores is set. Then the gain characteristics were studied using the model described in [4] through iteration till the absolute gain and gain fluctuations could be obtained close to the target values.

\section{RESULTS AND DISCUSSIONS}

With the optimized parameters obtained through the procedure outlined in section 2, a sample of calculated gain spectrum of the proposed ATC fiber for one set of design parameters is shown in Fig. 4. As can be seen from this figure, the gain peak around $1530 \mathrm{~nm}$ goes down because of the filtering action of the fiber at selective wavelengths around $1530 \mathrm{~nm}$. The figure shows a median gain of $\sim 38 \mathrm{~dB}$ with a gain ripple within $\pm 1 \mathrm{~dB}$ within the C-band. Corresponding noise figure variation with wavelength is shown in Fig. 5. It can be seen from this figure that the noise figure improves at longer wavelengths. This is due to enhanced overlap of pump and signal powers in the doped region at longer wavelengths. We have also made a detailed tolerance study as to different fiber parameters from the point of view of any detrimental effect on the flattened gain spectrum.

Our study revealed that control of the un-doped core parameters is very critical. With so obtained simulation studies and taking in to account limitations of the MCVD fabrication facility available with us, we redesigned the ATC for realizing an EDFA with medium gain of $\sim 20 \mathrm{~dB}$ with gain excursions below $\pm 1.5 \mathrm{~dB}$. With the so designed nominal parameters for the ATC as initial parameters to define the fabrication recipe, two independent fiber preforms were fabricated; core 1 


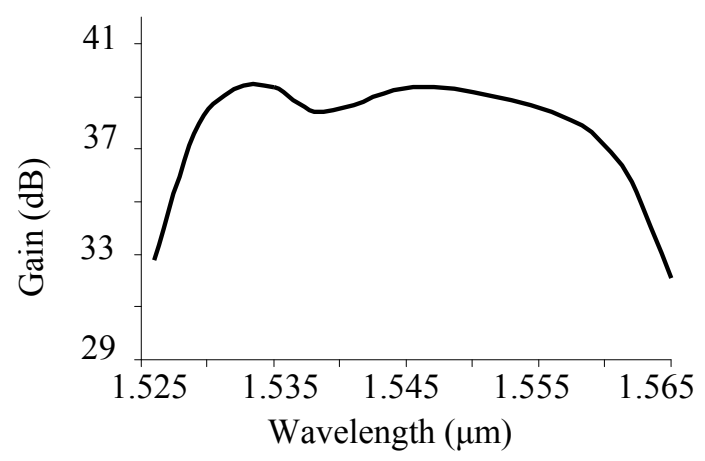

Fig. 4: Gain spectrum of the designed twin core EDFA.

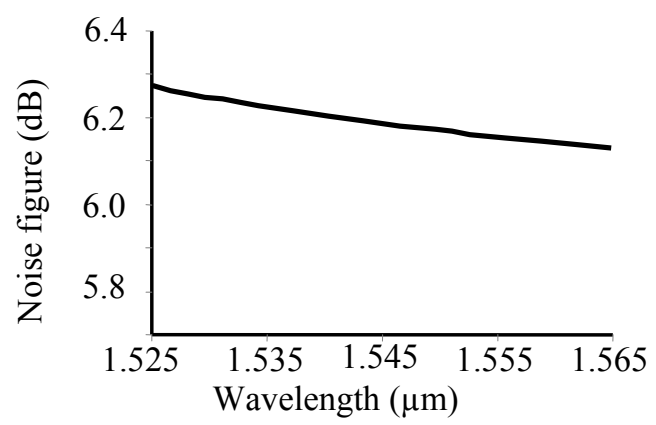

Fig. 5: Noise figure of proposed ATC based EDFA.

was solution-doped with $\mathrm{Er}^{3+}$ ions and core 2 was a relatively highly doped with germania. These were then selectively polished for achieving a target separation between the two cores at the fiber stage and assembled. The assembled polished fiber preforms were then drawn into a twin core fiber purported to function as an ATC fiber directional coupler. The optical micrograph and the refractive index profile along the axis going through centres of both cores of the fabricated ATC fiber is shown in Fig. 6. The core diameter (and core-cladding index difference) of the Er-doped and undoped core are $8.5 \mu \mathrm{m}(\Delta n=0.005)$ and $2.1 \mu \mathrm{m}(\Delta n=0.021)$, respectively. This fabricated ATC fiber has a core separation equal to $11 \mu \mathrm{m}$.

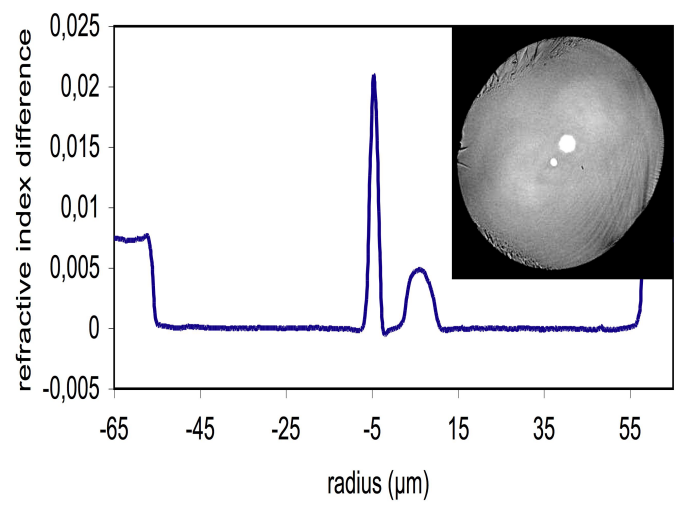

Fig. 6: Measured RIP of the twin core fiber; the inset shows optical micrograph of the ATC fiber, showing the un-doped core (smaller) and the Er-doped core (larger). 
A preliminary proof of light coupling from one core to the other is shown in Fig. 7. The amplified spontaneous emission (ASE) generated in the Er-doped core by 980-nm pumping is collected from both cores at the output end. This figure clearly illustrates a wavelength-sensitive share of the ASE. It can also be seen that the typical $1530 \mathrm{~nm}$ emission peak is filtered out to the undoped core output. Detailed experimental study of gain characteristics of the fabricated fiber is underway and will be reported elsewhere.

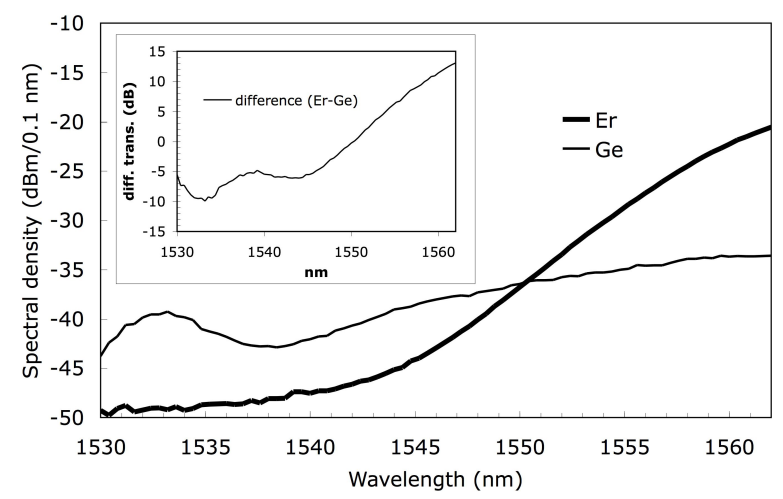

Fig. 7: Amplified spontaneous emission under 980-nm pumping, at the output end of the Er-doped (thick) and un-doped cores (thin). Inset: difference between Er- and undoped cores.

\section{CONCLUSIONS}

We have proposed an inherently gain flattened fiber amplifier using asymmetric twin core fiber in which one of the cores is doped with $\mathrm{Er}^{3+}$ while other core is un-doped. The proposed design has a theoretical gain spectrum flattened within $\sim \pm$ $1 \mathrm{~dB}$ over $32 \mathrm{~nm}$ of bandwidth (from $1530 \mathrm{~nm}$ to $1562 \mathrm{~nm}$ ). By keeping the tolerances of MCVD fabrication process in mind, we then targeted for a design having $20 \mathrm{~dB}$ gain with gain fluctuations within $\pm 1.5 \mathrm{~dB}$. We then fabricated the proposed asymmetric twin core fiber by polishing and assembling two independent preforms and drawing the assembly. Preliminary characterization results show the ASE peak filtering through power coupling from Er-doped core to the undoped core. Such an intrinsically gain flattened EDFA should be attractive for reduced cost-driven transparent metro networks as it would cut down the cost on gain equalization filter head of EDFAs required for such networks.

\section{REFERENCES}

[1] Nagaraju, B., Paul, M. C., Pal, M., Pal, A., Varshney, R. K., Pal, B. P., Bhadra, S. K., Monnom, G. and Dussardier, B., "Design and fabrication of an intrinsically gain flattened Erbium doped fiber amplifier", Opt. Commun., $282, .2335-2338$ (2009).

[2] Laming, R. I., Minelly,J. D., Dong, L., and Zervas,M. N., "Twin-core erbium doped fiber amplifier with passive spectral gain equalization", Electron. Lett., 29, 509-510 (1993).

[3] Wu, B., and Chu, P.L., "A twin-core erbium-doped fiber amplifier", Opt. Commun., 110, 545-548 (1994).

[4] Pedersen, B., "Small-signal erbium-doped fiber amplifiers pumped at $980 \mathrm{~nm}$ : a design study", Opt. Quant. Electron., 26, S273-S284 (1994).

[5] Snyder, A. W., and Ankiewicz, A., "Optical Fiber Couplers-Optimum Solution for Unequal Cores", J. Lightwave Tech., 6, 463-474 (1988). 